\title{
Planning Horizon and Its Effects on CBM System
}

\author{
${ }^{1}$ Akpan W. A \\ ${ }^{1}$ Mechanical and Aerospace \\ Engineering Department, University \\ of Uyo
}

\author{
${ }^{2}$ Odukwe A. O \\ ${ }^{2}$ Mechanical Engineering \\ Department University of Nigeria \\ Nsukka
}

\author{
${ }^{3}$ Enefiok O. U \\ ${ }^{3}$ Mechanical and Aerospace \\ Engineering Department, University \\ of Uyo
}

\begin{abstract}
This study investigates a problem of machine inspection and condition monitoring (CM) for condition based maintenance (CBM) in the Nigerian electric power industry using one and two years as planning horizons. These planning horizons were applied on a developed model and prescribed method of solution of combinatorial optimization to obtain results. A two years planning horizon provides a savings of $10 \%, 16 \%$ and $27 \%$ respectively compared to a one year planning horizon for one month, two months and three months inspection intervals. Increasing the planning horizon has the tendency to decrease the CBM total expected cost of the system. Implementation of this strategy stage by stage can provide an optimum planning horizon for the system during its useful life.
\end{abstract}

Keywords: CBM, Planning Horizon, maintenance scheduling, CM.

\section{INTRODUCTION}

Over the decades, the Nigerian Electric Power Industry has evolved tremendously. In recent times, it has shown the indication of moving from a regulated enterprise to a deregulated one. However, amount of electric power produced in the last five years has always been below 5,000 MW. With a population of over 180 million people, this amount of electricity is crossly inadequate. The promised made by various government to provide an uninterrupted power supply of electricity to its citizens has failed. Providing 24 hours of electricity daily to Nigerians should be taken as yardstick to measure the performance of any government in Nigeria. The supply of uninterruptable electricity is a figure of merit that measures the level of development of any country. With the concerted effort put up by government in its anti-corruption war and the drive for industrialization, much is still desired to show its reflection in the amount of power available to its citizens. Many companies have closed shops and the remaining few are still struggling to survive.

Proper management of the electricity industry of the country is needed. Deregulation is the key. Installation, operation and maintenance of the facilities in this sector should be the central focus.

The use of condition based maintenance will accelerate and fasten growth in the Nigerian electric power industry.

Condition based maintenance (CBM) is an equipment maintenance procedure for detecting the condition of the equipment in order to evaluate whether it will fail during some future period and acting appropriately to avoid the consequence of that failure [1]. Maintenance is carried out when there is an obvious need which will increase the availability of the equipment in the system, as well as lower the maintenance cost. The data acquired could be used to determine whether the system is running at a normal operating condition. If the limits of the preset values are exceeded, the reason behind it can be determined and prediction made for future equipment breakdown and failure. Conditioned based maintenance requires taking the result of the analysis and planning the maintenance afterwards. A structure is needed for effective utilization and communication of condition monitoring data within the establishment requires [2].

Condition monitoring is important to the maintenance personnel as it allows it plan preventive work and possible serious consequences of breakdown [3].CM has the capacity to reduce maintenance cost by 45\% [4]. The typical purpose of condition monitoring is to identify potential failure with the intension to accurately schedule maintenance activity so as to prevent operational interruption [5].

These warnings are known as potential failures [6]. It is identifiable physical conditions which indicate that a functional failure is about to occur or is in the process of occurring.

Akpan, et al; 2016 presented and solved a CBM problem for a system subject to inspection.

The objective of this work is to investigate the effects of planning horizon on CBM total expected cost (TEC) and to present and solve a formulated combinatorial optimization problem for a four machine system based on two planning horizons of one and two years.

\section{METHODOLOGY}

Four turbines in Afam (IV) electric power station in Nigeria were used in the case study. Data were obtained from this source and elaborate discussions were made with managers, supervisors, engineers and maintainers on CBM implementation strategy in the organization. This data was applied on a developed model and combinatorial optimization used as a method of solution.

In this study an exponential distribution was assumed.

$$
f_{i}(t)=\lambda_{i} e^{-\lambda_{i} t}
$$

where $\lambda_{i}$ is the failure rate of the CM component in machine $\mathrm{i}$. 
where $n_{f i}$ the actual is number of failure of machine $\mathrm{i}$ in a planning horizon.

$\lambda_{i}=\frac{1}{M T B F_{n i}}$

where the mean time to failure is given as

$\operatorname{MBTF}_{n i}=\frac{U_{T}}{n_{f i}}$

$U_{T}$ is the uptime of machine $\mathrm{i}$

The cumulative distribution function $(\mathrm{CDF})$ is given as:

$\bar{F}\left(t_{i j}\right)=1-F_{i, j}(t)$

For an exponential distribution,

$F_{i, j}(t)=\int f_{i}(t) d t$

The CBM model had been presented and solved by Akpan, et al; [7].

$T E C=A_{v}+\sum_{i=1}^{N} \frac{T}{T_{i}} a_{i}+\sum_{i=1}^{N} \sum_{j=o}^{n-1_{i}} C_{i d i, j+1}+\sum_{i=1}^{N} \sum_{j=0}^{n-1_{i}} C_{i, j+1}$

where TEC is the total expected cost of the system

And,

$$
C P T=\frac{A_{v}}{T}+\sum_{i=1}^{N} \frac{a_{i}}{T_{i}}+\sum_{i=1}^{N} \sum_{j=0}^{n_{i}-1} \frac{C_{i d i, j+1}}{T}+\sum_{i=1}^{N} \sum_{j=0}^{n-1_{i}} \frac{C_{i, j+1}}{T}
$$

CPT is the average cost per month

$A_{v}, a_{i}, C_{i d i, j+1}$ and $C_{i, j+1}$ are given below:

$$
A_{v}=P / n_{m}
$$

$A_{v}$ is the depreciation cost, $\mathrm{P}$ is the acquisition cost, $n_{m}$ the planned years of replacement

$a_{i}=C_{L} t_{\text {inspect }}{ }_{\mathrm{i}, \mathrm{j}}$

$a_{i}$ is the inspection cost of machine $\mathrm{i}, C_{L}$ labour cost and $t_{\text {inspect }}$, time to inspect machine $\mathrm{i}$

$C_{i d i, j+1}=P_{L} t_{d m i}$

$C_{i d i, j+1}$ is the down time cost of machine $\mathrm{i}$ in interval $\mathrm{j}, P_{L}$ is the production loss and $t_{d m i}$, the down time for production loss.

The failure cost $C_{i, j+1}$ is expressed as:

$C_{i, j+!}=\frac{r_{i}\left(1-e^{-\lambda_{i} t_{i, j+!}}\right)+s_{i}\left(t_{i, j+!}\right)+s_{i} t_{i, j+1} e^{-\lambda_{i} t_{i, j+1}}-\frac{s_{i}}{\lambda_{i}}\left(1-e^{-\lambda_{i} t_{i, J+1}}\right)}{e^{-\lambda_{i} t_{i, j+!}}}$

where:

$r_{i}$ is the repair cost, $s_{i}$ the increased cost of running the machine above alarm limit,$t_{i, j+1}$ the time interval

A combinatorial optimization technique was used as a method of solution in this research.

The model formulation is:

Minimize the CBM cost (TEC)

$T E C=A_{v}+\sum_{i=1}^{N} \frac{T}{T_{i}} a_{i}+\sum_{i=1}^{N} \sum_{j=o}^{n-1_{i}} C_{i d i, j+1}+\sum_{i=1}^{N} \sum_{j=0}^{n-1_{i}} C_{i, j+1}$

Subject to

$T_{i}=K_{i} T_{o}$

$A_{v}, a_{i}, C_{i d i, j+1}$ and $C_{i, j+1}$ are given as in equations 8 to 11 . 
$K_{i}$ is an integer, $T_{o}$ is the basic cycle, $T_{i}$ is the inspection interval of machine i. Further possible combinations of $T_{i}$ are generated with multiple of $T_{o}$, e.g. $2 T_{0}$ and $3 T_{0}$.

The unit has four turbines as shown in Table one below.

The planning horizons are one and two years respectively $(T=1$ year and 2 years).

Table one shows the input data for Afam (IV) Electric Power Station.

Table 1: Input Data for Afam (IV) Electric Power Station.

Machine Machine type $\lambda_{i}$ (per year) $r_{i}(\mathbf{N}) s_{i}=25 \% r_{i}(\mathbf{N}) \quad a_{1} \mathbf{N} A_{v}=17,715,760(\mathbf{N})$

\begin{tabular}{llllll}
\hline 1 & Gas turbine & 1.2 & 217741682 & 54435420 & 300000 \\
2 & Gas turbine & 1.2 & 217741682 & 54435420 & 300000 \\
3 & Gas turbine & 1.2 & 217741682 & 54435420 & 300000 \\
4 & Gas turbine & 1.2 & 217741682 & 54435420 & 300000 \\
\hline
\end{tabular}

* $A_{v}$ is CM instrument cost for the system.

III RESULTS AND DISCUSSION

Table two shows the total expected cost for a one year planning horizon for the plant.

Table 2: TEC Cost and Various Combinations at Afam (IV) for one year planning horizon

\begin{tabular}{|c|c|c|c|}
\hline $\mathbf{S} / \mathbf{N}$ & Combinations & Total Expected Cost $(\mathrm{N})$ & $\begin{array}{l}\text { Average Cost per Month } \\
\text { (N) }\end{array}$ \\
\hline 1 & $T_{1}=T_{2}=T_{3}=T_{4}=T_{0}$ & $11,959,027,478.48$ & $996,585,623.21$ \\
\hline 2 & $T_{1}=T_{2}=T_{3}=T_{4}=2 T_{0}$ & $6,603,344,243.89$ & $550,278,686.99$ \\
\hline 3 & $T_{1}=T_{2}=T_{3}=T_{4}=3 T_{0}$ & $4,833,239,016.77$ & $402,769,918.06$ \\
\hline 4 & $T_{1}=2 T_{0} \quad T_{2}=T_{3}=T_{4}=T_{0}$ & $10,620,106,669.831$ & $885,008,889.15$ \\
\hline 5 & $T_{2}=2 T_{0} T_{1}=T_{3}=T_{4}=T_{0}$ & $10,620,106,669.831$ & $885,008,889.15$ \\
\hline 6 & $T_{3}=2 T_{0} \quad T_{1}=T_{2}=T_{4}=T_{0}$ & $10,620,106,669.831$ & $885,008,889.15$ \\
\hline 7 & $T_{4}=2 T_{0}, T_{1}=T_{2}=T_{3}=T_{0}$ & $10,620,106,669.831$ & $885,008,889.15$ \\
\hline 8 & $T_{1}=T_{2}=2 T_{0} \quad T_{3}=T_{4}=T_{0}$ & $9,281,185,861.185$ & $773,432,155.10$ \\
\hline 9 & $T_{1}=T_{3}=2 T_{0}, T_{2}=T_{4}=T_{0}$ & $9,281,185,861.185$ & $773,432,155.10$ \\
\hline 10 & $T_{1}=T_{4}=2 T_{0}, T_{2}=T_{3}=T_{0}$ & $9,281,185,861.185$ & $773,432,155.10$ \\
\hline 11 & $T_{2}=T_{3}=2 T_{0}, T_{1}=T_{4}=T_{0}$ & $9,281,185,861.185$ & $773,432,155.10$ \\
\hline 12 & $T_{2}=T_{4}=2 T_{0}, T_{1}=T_{3}=T_{0}$ & $9,281,185,861.185$ & $773,432,155.10$ \\
\hline 13 & $T_{3}=T_{4}=2 T_{0}, T_{1}=T_{2}=T_{0}$ & $9,281,185,861.185$ & $773,432,155.10$ \\
\hline 14 & $T_{1}=T_{2}=T_{3}=2 T_{0}, T_{4}=T_{0}$ & $7,942,265,052.540$ & $661,855,421.05$ \\
\hline 15 & $T_{1}=T_{2}=T_{4}=2 T_{0}, T_{3}=T_{0}$ & $7,942,265,052.540$ & $661,855,421.05$ \\
\hline 16 & $T_{2}=T_{3}=T_{4}=2 T_{0}, T_{1}=T_{0}$ & $7,942,265,052.540$ & $661,855,421.05$ \\
\hline 17 & $T_{3}=T_{1}=T_{4}=2 T_{0}, T_{2}=T_{0}$ & $7,942,265,052.540$ & $661,855,421.05$ \\
\hline 18 & $T_{1}=3 T_{0}, T_{2}=T_{3}=T_{4}=T_{0}$ & $10,177,580,363.049$ & $848,131,696.92$ \\
\hline 19 & $T_{2}=3 T_{0}, T_{1}=T_{3}=T_{4}=T_{0}$ & $10,177,580,363.049$ & $848,131,696.92$ \\
\hline 20 & $T_{3}=3 T_{0}, T_{1}=T_{2}=T_{3}=T_{0}$ & $10,177,580,363.049$ & $848,131,696.92$ \\
\hline 21 & $T_{4}=3 T_{0}, T_{1}=T_{2}=T_{3}=T_{0}$ & $10,177,580,363.049$ & $848,131,696.92$ \\
\hline 22 & $T_{1}=T_{2}=3 T_{0}, T_{3}=T_{4}=T_{0}$ & $8,396,133,247.623$ & $699,677,770.64$ \\
\hline 23 & $T_{1}=T_{3}=3 T_{0}, T_{2}=T_{4}=T_{0}$ & $8,396,133,247.623$ & $699,677,770.64$ \\
\hline
\end{tabular}




\begin{tabular}{|c|c|c|c|}
\hline 24 & $T_{1}=T_{4}=3 T_{0}, T_{2}=T_{3}=T_{0}$ & $8,396,133,247.623$ & $699,677,770.64$ \\
\hline 25 & $T_{2}=T_{4}=3 T_{0}, T_{1}=T_{3}=T_{0}$ & $8,396,133,247.623$ & $699,677,770.64$ \\
\hline 26 & $T_{2}=T_{3}=3 T_{0}, T_{1}=T_{4}=T_{0}$ & $8,396,133,247.623$ & $699,677,770.64$ \\
\hline 27 & $T_{3}=T_{4}=3 T_{0}, T_{1}=T_{2}=T_{0}$ & $8,396,133,247.623$ & $699,677,770.64$ \\
\hline 28 & $T_{1}=T_{2}=T_{3}=3 T_{0}, T_{4}=T_{0}$ & $6,614,686,132.196$ & $551,223,844.35$ \\
\hline 29 & $T_{1}=T_{2}=T_{4}=3 T_{0}, T_{3}=T_{0}$ & $6,614,686,132.196$ & $551,223,844.35$ \\
\hline 30 & $T_{2}=T_{3}=T_{4}=3 T_{0}, T_{1}=T_{0}$ & $6,614,686,132.196$ & $551,223,844.35$ \\
\hline 31 & $T_{3}=T_{1}=T_{4}=3 T_{0}, T_{2}=T_{0}$ & $6,614,686,132.196$ & $551,223,844.35$ \\
\hline 32 & $T_{1}=2 T_{0}, T_{2}=T_{3}=T_{4}=3 T_{0}$ & $5,275,765,677.993$ & $439,647,139.83$ \\
\hline 33 & $T_{2}=2 T_{0}, T_{1}=T_{2}=T_{4}=3 T_{0}$ & $5,275,765,677.993$ & $439,647,139.83$ \\
\hline 34 & $T_{3}=2 T_{0}, T_{1}=T_{3}=T_{4}=3 T_{0}$ & $5,275,765,677.993$ & $439,647,139.83$ \\
\hline 35 & $T_{4}=2 T_{0}, T_{1}=T_{2}=T_{3}=3 T_{0}$ & $5,275,765,323.550$ & $439,647,139.83$ \\
\hline 36 & $T_{1}=T_{2}=2 T_{0}, T_{3}=T_{4}=3 T_{0}$ & $5,718,291,630.331$ & $439,647,139.83$ \\
\hline 37 & $T_{1}=T_{3}=2 T_{0}, T_{2}=T_{4}=3 T_{0}$ & $5,718,291,630.331$ & $439,647,139.83$ \\
\hline 38 & $T_{1}=T_{4}=2 T_{0}, T_{2}=T_{3}=3 T_{0}$ & $5,718,291,630.331$ & $439,647,139.83$ \\
\hline 39 & $T_{2}=T_{3}=2 T_{0}, T_{1}=T_{4}=3 T_{0}$ & $5,718,291,630.331$ & $439,647,139.83$ \\
\hline 40 & $T_{2}=T_{4}=2 T_{0}, T_{1}=T_{3}=3 T_{0}$ & $5,718,291,630.331$ & $439,647,139.83$ \\
\hline 41 & $T_{3}=T_{4}=2 T_{0}, T_{1}=T_{2}=3 T_{0}$ & $6,160,817,937.113$ & $513,401,494.76$ \\
\hline 42 & $T_{1}=T_{2}=T_{3}=2 T_{0}, T_{4}=3 T_{0}$ & $6,160,817,937.113$ & $513,401,494.76$ \\
\hline 43 & $T_{1}=T_{2}=T_{4}=2 T_{0}, T_{3}=3 T_{0}$ & $6,160,817,937.113$ & $513,401,494.76$ \\
\hline 44 & $T_{2}=T_{3}=T_{4}=2 T_{0}, T_{1}=3 T_{0}$ & $6,160,817,937.113$ & $513,401,494.76$ \\
\hline 45 & $T_{3}=T_{1}=T_{4}=2 T_{0}, T_{2}=3 T_{0}$ & $6,160,817,937.113$ & $513,401,494.76$ \\
\hline 46 & $T_{1}=T_{0}, T_{2}=T_{3}=2 T_{0}, T_{4}=3 T_{0}$ & $7,499,739,250.431$ & $624,978,270.87$ \\
\hline 47 & $T_{1}=T_{0}, T_{2}=T_{4}=2 T_{0}, T_{3}=3 T_{0}$ & $7,499,738,745.758$ & $624,978,270.87$ \\
\hline 48 & $T_{1}=T_{0}, T_{3}=T_{4}=2 T_{0}, T_{1}=3 T_{0}$ & $7,499,738,745.758$ & $624,978,270.87$ \\
\hline 49 & $T_{2}=T_{0}, T_{3}=T_{4}=2 T_{0}, T_{1}=3 T_{0}$ & $7,499,738,745.758$ & $624,978,270.87$ \\
\hline 50 & $T_{2}=T_{0}, T_{2}=T_{4}=2 T_{0}, T_{3}=3 T_{0}$ & $7,499,738,745.758$ & $624,978,270.87$ \\
\hline 51 & $T_{3}=T_{0}, T_{1}=T_{2}=2 T_{0}, T_{4}=3 T_{0}$ & $7,499,738,745.758$ & $624,978,270.87$ \\
\hline 52 & $T_{3}=T_{0}, T_{2}=T_{4}=2 T_{0}, T_{1}=3 T_{0}$ & $7,499,738,745.758$ & $624,978,270.87$ \\
\hline 53 & $T_{4}=T_{0}, T_{1}=T_{2}=2 T_{0}, T_{3}=3 T_{0}$ & $7,499,738,745.758$ & $624,978,270.87$ \\
\hline 54 & $T_{4}=T_{0}, T_{2}=T_{3}=2 T_{0}, T_{1}=3 T_{0}$ & $7,499,738,745.758$ & $624,978,270.87$ \\
\hline
\end{tabular}

Table 3 shows the total expected cost for two years planning horizon for Afam (IV) electric power station.

Table 3: TEC Cost and Various Combinations at Afam (IV) for two years planning horizon.

\begin{tabular}{llll}
\hline $\mathbf{S} / \mathbf{N}$ & Combinations & Total Expected Cost $(\mathbf{N})$ & $\begin{array}{l}\text { Average Cost per Month } \\
(\mathbf{N})\end{array}$ \\
\hline 1 & $T_{1}=T_{2}=T_{3}=T_{4}=T_{0}$ & $22,704,831,220.60$ & $946,034,634.19$ \\
2 & $T_{1}=T_{2}=T_{3}=T_{4}=2 T_{0}$ & $12,118,530,668.73$ & $504,938,777.86$ \\
3 & $T_{1}=T_{2}=T_{3}=T_{4}=3 T_{0}$ & $8,384,784,048.35$ & $349,366,002.05$
\end{tabular}




\begin{tabular}{|c|c|c|c|}
\hline 4 & $T_{1}=2 T_{0} \quad T_{2}=T_{3}=T_{4}=T_{0}$ & $20,018,395,582.632$ & $834,099,815.94$ \\
\hline 5 & $T_{2}=2 T_{0} T_{1}=T_{3}=T_{4}=T_{0}$ & $20,018395582.632$ & $834,099,815.94$ \\
\hline 6 & $T_{3}=2 T_{0} \quad T_{1}=T_{2}=T_{4}=T_{0}$ & $20,018395582.632$ & $834,099,815.94$ \\
\hline 7 & $T_{4}=2 T_{0}, T_{1}=T_{2}=T_{3}=T_{0}$ & $20,018,395,582.632$ & $834,099,815.94$ \\
\hline 8 & $T_{1}=T_{2}=2 T_{0} \quad T_{3}=T_{4}=T_{0}$ & $17,331,959,944.665$ & $722,164,997.69$ \\
\hline 9 & $T_{1}=T_{3}=2 T_{0}, T_{2}=T_{4}=T_{0}$ & $17,331,959,944.665$ & $722,164,997.69$ \\
\hline 10 & $T_{1}=T_{4}=2 T_{0}, T_{2}=T_{3}=T_{0}$ & $17,331,959,944.665$ & $722,164,997.69$ \\
\hline 11 & $T_{2}=T_{3}=2 T_{0}, T_{1}=T_{4}=T_{0}$ & $17,331,959,944.665$ & $722,164,997.69$ \\
\hline 12 & $T_{2}=T_{4}=2 T_{0}, T_{1}=T_{3}=T_{0}$ & $17,331959944.665$ & $722,164,997.69$ \\
\hline 13 & $T_{3}=T_{4}=2 T_{0}, T_{1}=T_{2}=T_{0}$ & $17,331,959,944.665$ & $722,164,997.69$ \\
\hline 14 & $T_{1}=T_{2}=T_{3}=2 T_{0}, T_{4}=T_{0}$ & $14,645,524,306.699$ & $610,230,179.45$ \\
\hline 15 & $T_{1}=T_{2}=T_{4}=2 T_{0}, T_{3}=T_{0}$ & $14,645,524,306.699$ & $610,230,179.45$ \\
\hline 16 & $T_{2}=T_{3}=T_{4}=2 T_{0}, T_{1}=T_{0}$ & $14,645,524,306.699$ & $610,230,179.45$ \\
\hline 17 & $T_{3}=T_{1}=T_{4}=2 T_{0}, T_{2}=T_{0}$ & $14,645,524,306.699$ & $610,230,179.45$ \\
\hline 18 & $T_{1}=3 T_{0}, T_{2}=T_{3}=T_{4}=T_{0}$ & $19,124,819,427.536$ & $796,867,476.15$ \\
\hline 19 & $T_{2}=3 T_{0}, T_{1}=T_{3}=T_{4}=T_{0}$ & $19,124,819,427.536$ & $796,867,476.15$ \\
\hline 20 & $T_{3}=3 T_{0}, T_{1}=T_{2}=T_{3}=T_{0}$ & $19,124,819,427.536$ & $796,867,476.15$ \\
\hline 21 & $T_{4}=3 T_{0}, T_{1}=T_{2}=T_{3}=T_{0}$ & $19,124,819,427.536$ & $796,867,476.15$ \\
\hline 22 & $T_{1}=T_{2}=3 T_{0}, T_{3}=T_{4}=T_{0}$ & $15,544,807,634.473$ & $647,700,318.10$ \\
\hline 23 & $T_{1}=T_{3}=3 T_{0}, T_{2}=T_{4}=T_{0}$ & $15,544,807,634.473$ & $647,700,318.10$ \\
\hline 24 & $T_{1}=T_{4}=3 T_{0}, T_{2}=T_{3}=T_{0}$ & $15,544,807,634.473$ & $647,700,318.10$ \\
\hline 25 & $T_{2}=T_{4}=3 T_{0}, T_{1}=T_{3}=T_{0}$ & $15,544,807,634.473$ & $647,700,318.10$ \\
\hline 26 & $T_{2}=T_{3}=3 T_{0}, T_{1}=T_{4}=T_{0}$ & $15,544,807,634.473$ & $647,700,318.10$ \\
\hline 27 & $T_{3}=T_{4}=3 T_{0}, T_{1}=T_{2}=T_{0}$ & $15,544,807,634.473$ & $647,700,318.10$ \\
\hline 28 & $T_{1}=T_{2}=T_{3}=3 T_{0}, T_{4}=T_{0}$ & $11,964,795,841.410$ & $498,533,160.06$ \\
\hline 29 & $T_{1}=T_{2}=T_{4}=3 T_{0}, T_{3}=T_{0}$ & $11,964,795,841.410$ & $498,533,160.06$ \\
\hline 30 & $T_{2}=T_{3}=T_{4}=3 T_{0}, T_{1}=T_{0}$ & $11,964,795,841.410$ & $498,533,160.06$ \\
\hline 31 & $T_{3}=T_{1}=T_{4}=3 T_{0}, T_{2}=T_{0}$ & $11,964,795,841.410$ & $498,533,160.06$ \\
\hline 32 & $T_{1}=2 T_{0}, T_{2}=T_{3}=T_{4}=3 T_{0}$ & $9,437,802,203.443$ & $393,241,758.48$ \\
\hline 33 & $T_{2}=2 T_{0}, T_{1}=T_{2}=T_{4}=3 T_{0}$ & $9,437,802,203.443$ & $393,241,758.48$ \\
\hline 34 & $T_{3}=2 T_{0}, T_{1}=T_{3}=T_{4}=3 T_{0}$ & $9,437,802,203.443$ & $393,241,758.48$ \\
\hline 35 & $T_{4}=2 T_{0}, T_{1}=T_{2}=T_{3}=3 T_{0}$ & $9,437,802,203.443$ & $393,241,758.48$ \\
\hline 36 & $T_{1}=T_{2}=2 T_{0}, T_{3}=T_{4}=3 T_{0}$ & $10,331,378,358.540$ & $430,474,098.27$ \\
\hline 37 & $T_{1}=T_{3}=2 T_{0}, T_{2}=T_{4}=3 T_{0}$ & $10,331,378,358.540$ & $430,474,098.27$ \\
\hline 38 & $T_{1}=T_{4}=2 T_{0}, T_{2}=T_{3}=3 T_{0}$ & $10,331,378,358.540$ & $430,474,098.27$ \\
\hline 39 & $T_{2}=T_{3}=2 T_{0}, T_{1}=T_{4}=3 T_{0}$ & $10,331,378,358.540$ & $430,474,098.27$ \\
\hline 40 & $T_{2}=T_{4}=2 T_{0}, T_{1}=T_{3}=3 T_{0}$ & $10,331,378,358.540$ & $430,474,098.27$ \\
\hline 41 & $T_{3}=T_{4}=2 T_{0}, T_{1}=T_{2}=3 T_{0}$ & $10,331,378,358.540$ & $430,474,098.27$ \\
\hline
\end{tabular}




$\begin{array}{llll}42 & T_{1}=T_{2}=T_{3}=2 T_{0}, T_{4}=3 T_{0} & 11,224,954,513.636 & 467,706,438.07 \\ 43 & T_{1}=T_{2}=T_{4}=2 T_{0}, T_{3}=3 T_{0} & 11,224,954,513.636 & 467,706,438.07 \\ 44 & T_{2}=T_{3}=T_{4}=2 T_{0}, T_{1}=3 T_{0} & 11,224,954,513.636 & 467,706,438.07 \\ 45 & T_{3}=T_{1}=T_{4}=2 T_{0}, T_{2}=3 T_{0} & 11,224,954,513.636 & 467,706,438.07 \\ 46 & T_{1}=T_{0}, T_{2}=T_{3}=2 T_{0}, T_{4}=3 T_{0} & 13,751,948,151.603 & 572,997,839.65 \\ 48 & T_{1}=T_{0}, T_{2}=T_{4}=2 T_{0}, T_{3}=3 T_{0} & 13,751,948,151.603 & 572,997,839.65 \\ 49 & T_{1}=T_{0}, T_{3}=T_{4}=2 T_{0}, T_{1}=3 T_{0} & 13,751,948,151.603 & 572,997,839.65 \\ 50 & T_{2}=T_{0}, T_{3}=T_{4}=2 T_{0}, T_{1}=3 T_{0} & 13,751,948,151.603 & 572,997,839.65 \\ 51 & T_{2}=T_{0}, T_{2}=T_{4}=2 T_{0}, T_{3}=3 T_{0} & 13,751,948,151.603 & 572,997,839.65 \\ 52 & T_{3}=T_{0}, T_{1}=T_{2}=2 T_{0}, T_{4}=3 T_{0} & 13,751,948,151.603 & 572,997,839.65 \\ 53 & T_{3}=T_{0}, T_{2}=T_{4}=2 T_{0}, T_{1}=3 T_{0} & 13,751,948,151.603 & 572,997,839.65 \\ 54 & T_{4}=T_{0}, T_{1}=T_{2}=2 T_{0}, T_{3}=3 T_{0} & 13,751,948,151.603 & 572,997,839.65 \\ & T_{4}=T_{0}, T_{2}=T_{3}=2 T_{0}, T_{1}=3 T_{0} & 13,751,948,151.603 & 572,997,839.65\end{array}$

From Table 2 and Table 3, at Afam (IV) the total expected cost for one month, two months and three months inspection interval for a one year planning horizon were: $\mathrm{N} 11,959,027,478.48$; N 6,603,344,243.89 and N 4,833,239,016.77 Akpan etal. However, for a two years planning horizon, the total expected cost for one month, two months and three months inspection interval are: $\mathbb{N} 22,704,831,220.60, \mathbb{N} 12,118,530,668.73$ and $\mathbb{N}$ 8,384,784,048.35 representing an increase of $90 \%, 84 \%$ and $73 \%$ respectively. These reductions provide CBM cost savings. Table 3 shows the total expected CBM cost for various combinations at Afam (IV) for a planning horizon of two years. Option 3 in Table 3 has the least total expected cost of $\mathrm{N} 8,384,784,048.35$ per year, with an average cost per month of $\mathbb{3} 39,366,002.05$ for three months inspection interval.

\section{CONCLUSIONS}

A two years planning horizon provides a savings of $10 \%, 16 \%$ and $27 \%$ respectively compared to a one year planning horizon for one month, two months and three months inspection intervals. Increasing the planning horizon has the tendency to decrease the CBM total expected cost of the system. Implementation of this strategy stage by stage can provide an optimum planning horizon for the system during its useful life. Further research work should be conducted for systems subject to other failure distributions like gamma and weibull.

[1] Bengtsson, M. (2004) Condition Based Maintenance in Technical Systems. (Online serial) Available:http://www.idp.mdh.se/forskninj/amneri/productprocess/projetk/cbm/index - asp? Prpb 106 [June 16, 2013].
Evans, J. P. (2003) "Utilizing Advanced Monitoring and Diagnostics Technologies to Implement a Condition-based Maintenance Programme. (Online serial) Available: http://images.tdworldexpo.com /files/ 1104/MT6\%20-\%20-\%Evans.pdf. [June 10, 2013]

[3] Koboa-Aduma, B. (1991) "Maintenance Management of Small scale Industries", in the Proceedings of the International Conference Workshop on Engineering for Accelerated Rural Faculty of Engineering, University of Nigeria, Nsukka. 243

[4] Guerin, D. (1977) "Condition Monitoring" The Production Engineering. March pp. 88-91.

[5] Laakso, K. and Rosqvist, T. (2002) The Use of Condition Monitoring Information for Maintenance Planning and Decision -Making. VTT Industrial System, Finland.

[6] Moubray, J. (2002) An Introduction to Predictive Maintenance. New York: Industrial Press.

[7] W. A. Akpan. Odukwe, A.O; Okorie B.A: Condition Based Maintenance in Nigerian Electric Power Industry: International Journal of Science and Technology Research (ISSTR) Vol.6, Issue 92017 